\title{
Impact of Manufacturing Tolerances of Waveguide Power Dividers on the Quiet Zone Quality of a Plane Wave Generator
}

\author{
Rutger van Boeijen ${ }^{1,2}$, Andrés Alayón Glazunov ${ }^{1,3}$ and Robert Rehammar ${ }^{2,3}$
}

\begin{abstract}
The effects of manufacturing tolerances on the quiet zone quality of a plane wave generator have been investigated. Waveguide power dividers required in the passive power distribution network of a plane wave generator have been designed. Monte Carlo simulations of the S-parameters of the designed power splitters have been performed by varying the power dividers' dimensions within manufacturing tolerances assuming a normal distribution. Furthermore, the splitting ratios resulting from the randomization are assumed to be uniformly distributed as the input for evaluating the quiet zone by means of Monte Carlo simulations. Assuming a 20 by 20 squared cm quiet zone, it has been found that for the considered setup that: (i) the phase deviation in the quiet zone never exceeds $+/-4.79$ degrees, and (ii) for the amplitude deviation, never exceeds $+/-0.91 \mathrm{~dB}$ within a single simulated quiet zone.
\end{abstract}

\section{INTRODUCTION}

5G New Radio standard frequencies, i.e., FR2, occupy the $\mathrm{mm}$-wave band from $22.250-52.60 \mathrm{GHz}$, [1]. The devices operating at these frequencies will meet several new challenges, e.g., due to the increased complexity introduced by the use of beam steering and corresponding transceiver design. These devices require new over-the-air (OTA) testing due to the lack of connected interfaces. In most cases, the UE will be in the far-field from the base station. To emulate the far-field signal of the base station, a plane wave can be synthesized by a planar antenna array, among other techniques, by means of a plane wave generator (PWG). These planar arrays can be fully populated as in [2], or irregular as in [3], [4]. In [3], [4] the irregular arrays employ a uniform excitation of the elements, while for the fully populated array of [2] a linear tapering over the elements has been employed. In [2] it was found that a PWG can form an adequate QZ at a distance of $45 \mathrm{~cm}$ from the PWG. For example, for a QZ with an approximate size of $20 \times 20 \mathrm{~cm}^{2}$, the amplitude and angular deviations of $\pm 0.75 \mathrm{~dB}$ and $\pm 7.5^{\circ}$, respectively, can be achieved. In this work a QZ sizing of $20 \times 20 \mathrm{~cm}^{2}$ is assumed, where the results of [2] are re-evaluated for this QZ sizing. The PWG comprises a $2 \mathrm{D}$ array antenna with $32 \times 32$ elements with linear tapering at the array edges. The bandwidth of interest for the PWG is from $26.5-29.5 \mathrm{GHz}$. A corporate feed network was employed to distribute the signal to the antenna elements. For this purpose, a passive power distribution network (PDN) consisting of power dividers devised with unique splitting ratios where obtained.

\footnotetext{
${ }^{1}$ Department of Electrical Engineering, University of Twente, $7500 \mathrm{AE}$ Enschede, The Netherlands.

${ }^{2}$ Bluetest AB, Gothenburg, Sweden.

${ }^{3}$ Department of Electrical Engineering, Chalmers University of Technology, Gothenburg, Sweden.

${ }^{*}$ Corresponding author: rutger.vanboeijen@bluetest.se
}

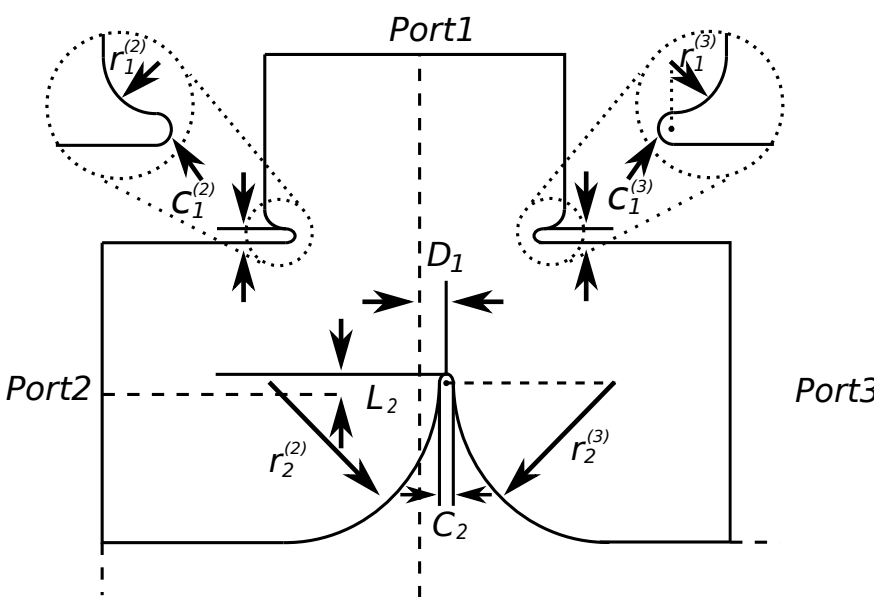

Fig. 1: Sketch of a waveguide power divider based on [6] and corresponding design parameters.

The effects of manufacturing tolerances of the power dividers on the generated quiet zone (QZ) of the PWG were not considered in [2]. Nor have similar results been published earlier to the best knowledge of the authors. Therefore, in this paper, in order to quantify the effects of non-ideal power dividers employed in the plane wave generator (PWG) of [2], these have been subjected to manufacturing tolerances. The power dividers are modelled as milled waveguides in CST and each is subjected to randomization by means of Monte Carlo (MC) simulations. In the MC simulation the variables are assumed to follow a normal distribution with a three-sigma value $3 \sigma=0.05 \mathrm{~mm}$, where $0.05 \mathrm{~mm}$ is the finest tolerance for linear dimensions up to $6 \mathrm{~mm}$ according to the DIN ISO 2768 standard.

\section{PASSIVE POWER DISTRIBUTION NETWORK}

In order to deliver the input signal to all the antenna elements at the right level, a power distribution network (PDN) is required. The considered passive PDN consists of $N$ layers of $1: 2$ power dividers, where $N=\log _{2}\left(N_{a}\right)$ and $N_{a}$ is the number of antenna elements. The array consists of $32 \times 32$ elements, hence 10 layers of power splitters are required. Since the PDN is large, the power dividers should have a low reflection coefficient. Indeed, with a reflection coefficient $|\Gamma|^{2}=0.001$, the reflected power would be approximately $1 \%$ of the input power (assuming that all reflected power is returned to the input). A reflection coefficient $|\Gamma|^{2}=0.001$ translates to $\left|S_{11}\right|=-30 \mathrm{~dB}$ for the power dividers over the entire bandwidth. The eight splitting ratios required to create the linear tapering obtained in [5] are $\{1: 1,1: 1.196,1$ : 
TABLE I: Dimensions of the waveguide power dividers.

\begin{tabular}{c|cccccc}
\hline \hline & $\mathbf{C}_{\mathbf{1}}$ & $\mathbf{C}_{\mathbf{2}}$ & $\mathbf{D}_{\mathbf{1}}$ & $\mathbf{L}_{\mathbf{2}}$ & $\mathbf{r}_{\mathbf{1}}$ & $\mathbf{r}_{\mathbf{2}}$ \\
\hline $\mathbf{1 : 1}$ (red) & 0.1575 & 0.5 & 0 & 0.2 & 0.5 & 2.6 \\
\hline $\mathbf{1 : 1 . 1 9 6}$ (grey) & 0.1575 & 0.5 & 0.14 & -0.14 & 0.5 & 2.5 \\
\hline $\mathbf{1 : 1 . 4 8 7}$ (yellow) & 0.1575 & 0.5693 & 0.33 & -0.14 & 0.5 & 2.5 \\
\hline $\mathbf{1 : 1 . 8 2 6}$ (green) & 0.1575 & 0.5693 & 0.5 & -0.2 & 0.5 & 2.5 \\
\hline $\mathbf{1 : 1 . 9 3 6}$ (blue) & 0.1575 & 0.5 & 0.57 & -0.2 & 0.5 & 2.5 \\
\hline $\mathbf{1 : 5 . 1 7 5}$ (cyan) & 0.1575 & 1 & 1.25 & -0.1 & 0.5 & 4 \\
\hline $\mathbf{1 : 7 . 0 2 7}$ (purple) & 0.1575 & 1.68 & 1.47 & -0.2 & 0.5 & 3.75 \\
\hline $\mathbf{1 : 7 . 8 4}$ (orange) & 0.1575 & 1.68 & 1.57 & -0.2 & 0.5 & 4 \\
\hline \hline
\end{tabular}

$1.487,1: 1.826,1: 1.936,1: 5.175,1: 7.027,1: 7.84\}$. In this paper we will use the same values in our investigations.

\section{A. Waveguide PDN design}

All the waveguides have been designed according to the standard WR-28 sizing. The dividers consist of a wedge and diaphragms following the design rules presented in [6]. A schematic representation of the power divider with corresponding design parameters are shown in Fig. 1. The parameters for the splitting ratios below $1: 3$ are determined using the design equations of [6]. These are then optimized to meet the $S_{11}$ requirement of the ideal splitting ratio at the design frequency, $f_{d}=28 \mathrm{GHz}$. The power dividers with splitting ratios larger than $1: 3$ are first designed by choosing $D_{1}$ satisfying the target splitting ratio. Afterwards they are further optimized to meet the $S_{11}$ requirement. Table I shows the final values of the design parameters of the power dividers.

The $S_{11}, S_{21}$ and $S_{31}$ of the designed power splitters are shown in Figs. 2a, 2b and 2c, respectively. Some of the power dividers do not meet the $S_{11}$ requirement over the entire bandwidth, especially the $1: 1.936$ power divider has a small impedance bandwidth defined at $\left|S_{11}\right|=-30 \mathrm{~dB}$. Hence, it can still be optimized further, but was deemed good enough for the current use case since $S_{11}, S_{21}$ and $S_{31}$ meet the requirements at the design frequency $f_{d}=28 \mathrm{GHz}$. Furthermore, it is worthwhile noting by inspection of the abovementioned figures that for all the simulated splitting ratios, the $S_{21}$-parameters slightly increase with frequency crossing the ideal (target) splitting ratio line at $f_{d}$. On the other hand, the opposite trend is observed for the $S_{31}$-parameters, i.e., it increases while also crossing the splitting ratio line at $f_{d}$.

\section{B. Manufacturing tolerance impact on S-parameters}

In this section we show the impact of the manufacturing tolerance on the designed power splitters. Manufacturing tolerances will lead to a variation of the resulting power splitting ratios deviating from the ideal values (these are the target design values shown in the first column of Table I). The varied parameters are shown in Figure 1. The variation of the parameters follows a Monte Carlo simulation procedure where the parameters are drawn from a normal distribution with a three-sigma value $3 \sigma=0.05 \mathrm{~mm}$, where $0.05 \mathrm{~mm}$ is the finest tolerance for linear dimensions up to $6 \mathrm{~mm}$ according to the DIN ISO 2768 standard. The mean values are given in Table I. A 100 different values of each design parameter
TABLE II: Dynamic range of the $S_{11}$-parameter and the percentage of cases not meeting the $S_{11}$ requirement.

\begin{tabular}{c|cc|cc|cc}
\hline \hline & \multicolumn{2}{|c|}{$\mathbf{2 6 . 5 ~ G H z}$} & \multicolumn{2}{c|}{$\mathbf{2 8 ~ G H z}$} & \multicolumn{2}{c}{$\mathbf{2 9 . 5} \mathbf{~ G H z}$} \\
\cline { 2 - 7 } & DR $[\mathrm{dB}]$ & $\%$ & DR $[\mathrm{dB}]$ & $\%$ & DR $[\mathrm{dB}]$ & $\%$ \\
\hline $\mathbf{1 : 1}$ & 0.38 & 1 & -0.15 & 0 & 2.92 & 28 \\
\hline $\mathbf{1 : 1 . 1 9 6}$ & 5.1 & 62 & -0.58 & 0 & -1.03 & 0 \\
\hline $\mathbf{1 : 1 . 4 8 7}$ & 6.0 & 90 & 1.36 & 2 & 1.36 & 5 \\
\hline $\mathbf{1 : 1 . 8 2 6}$ & 2.55 & 44 & -0.44 & 0 & 0.87 & 12 \\
\hline $\mathbf{1 : 1 . 9 3 6}$ & 5.0 & 94 & 2.94 & 42 & 3.2 & 46 \\
\hline $\mathbf{1 : 5 . 1 7 5}$ & 4.22 & 52 & 0.22 & 1 & 2.73 & 33 \\
\hline $\mathbf{1 : 7 . 0 2 7}$ & 3.42 & 48 & -3.27 & 0 & 2.85 & 42 \\
\hline $\mathbf{1 : 7 . 7 4}$ & 2.48 & 22 & -2.28 & 0 & 2.36 & 43 \\
\hline \hline
\end{tabular}

were randomly and independently generated resulting in 100 random values of the $S$-parameters, e.g., power splitting values for each ideal (target) power splitting values.

Figs. $2 \mathrm{~d}$ and $2 \mathrm{~h}$ show the effect of the random variations on the $S_{11}$ parameters of the $1: 1$ and $1: 7.84$ power dividers respectively. In order to quantify the deviation, the dynamic range (DR) is calculated as

$$
\mathrm{DR}=\operatorname{MAX}\left(20 \log \left(S_{11}\right)+30\right),
$$

where 30 appears since we are interested in the value relative the performance goal, i.e., $S_{11} \leq-30 \mathrm{~dB}$. The DR is shown in Table II along with the percentage of times when DR $>0 \mathrm{~dB}$ at 26.5-, 28- and $29.5 \mathrm{GHz}$. As can be seen from the table, the percentages are the highest at $26.5 \mathrm{GHz}$ for six of the eight power dividers. This is attributed to the designs having their best performance at frequencies higher than $28 \mathrm{GHz}$ as shown in Fig. 2a. The reverse is found for the designs where the best performance is below $28 \mathrm{GHz}$, e.g., as for the $1: 1$ power divider.

The variation of the $S_{21}$ and $S_{31}$ parameters are shown in Figs. 2e and $2 \mathrm{f}$, respectively for the $1: 1$ power divider. The corresponding figures for the $1: 7.84$ power divider are given in Figs. $2 \mathrm{~h}$ and $2 \mathrm{i}$, respectively.

\section{QUIET ZONE QUALITY EVALUATION}

The power and phase in the quite zone (QZ) using ideal elements are shown in Figs. 3a and 3d, respectively. These results were obtained assuming the ideal splitting ratios.

However, as we have seen from the above section, manufacturing tolerances induce a variation of the $|S|$-parameters of the power dividers. Therefore, the evaluation of the impact of manufacturing tolerances of waveguide power dividers on the QZ of the PWG is required.

To generate a QZ subjected to variations due to manufacturing tolerances, each ideal power divider is replaced by a non-ideal power divider, i.e., the ones presented above. First, the ideal (target) splitting ratio of the power divider is determined. For each the splitting ratios, a set containing 100 non-ideal power dividers exists, which $S$-parameters have been generated by the Monte Carlo simulations described above. Since we have now 100 different realizations of each power splitting ratio altered by the manufacturing tolerances, we can then characterize their combined impact on the QZ quality. For this purpose, a power divider and corresponding 


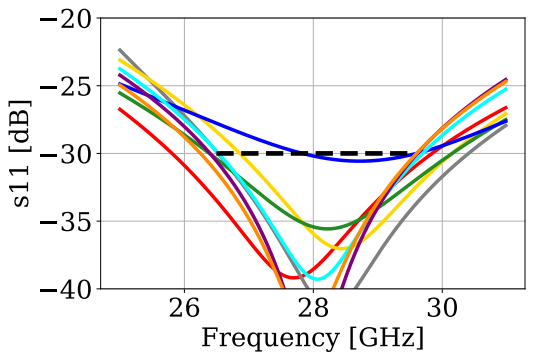

(a)

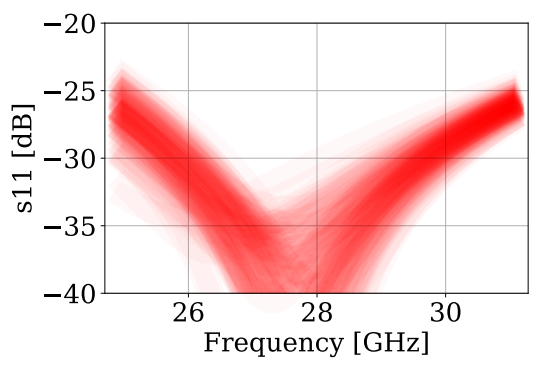

(d)

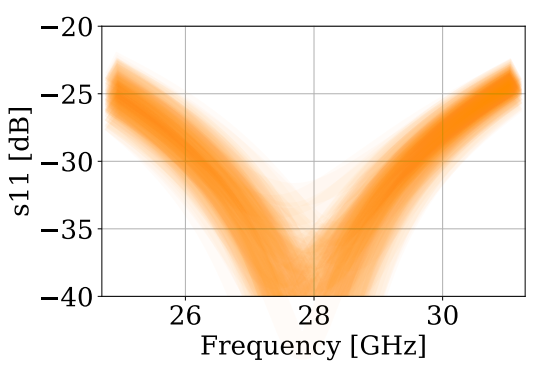

(g)

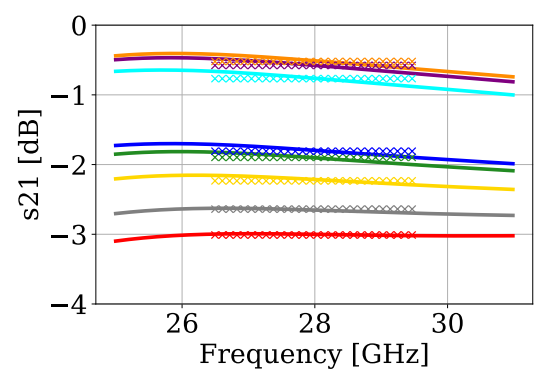

(b)

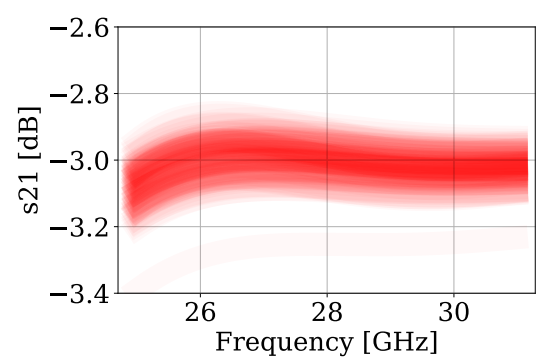

(e)

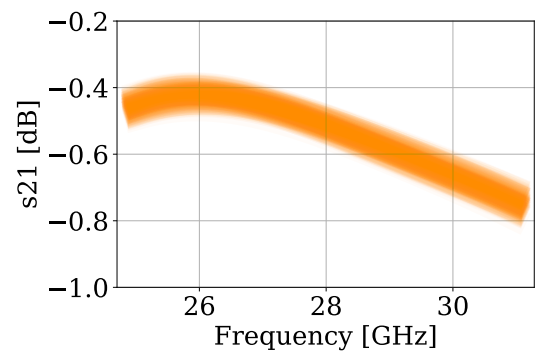

(h)

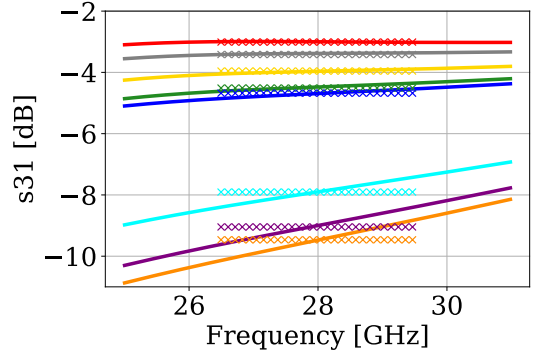

(c)

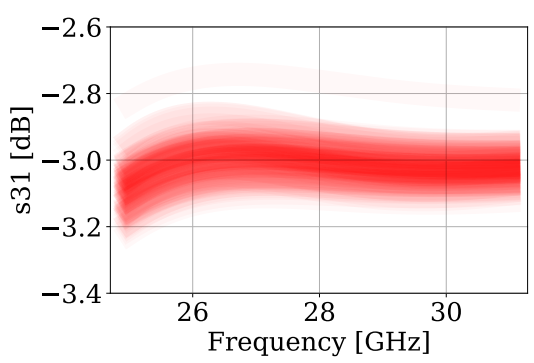

(f)

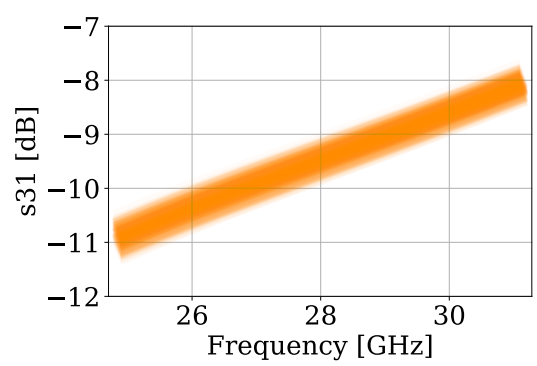

(i)

Fig. 2: The $S$-parameters of the designed power dividers are shown for (a) the $S_{11}$-parameters, where the black dashed line denotes the target value over the bandwidth of interest, (b) the $S_{21}$-parameters, and (c) the $S_{31}$-parameters with cross markers indicating the corresponding parameters for ideal splitting ratios. The $S$-parameters for the $1: 1$ power divider resulting from the MC simulations are shown for (d) the $S_{11}$-parameters, (e) the $S_{21}$-parameters and (f) the $S_{31}$-parameters. Corresponding results for the $1: 7.84$ power divider are shown for $(\mathrm{g})$ the $S_{11}$-parameters, (h) the $S_{21}$-parameters and i the $S_{31}$-parameters. All colors are according to the code provided in Table I.

$S$-parameters are chosen randomly from the uniform integer number distribution $\mathrm{U}\left(0, N_{s}-1\right)$, where $N_{s}=100$. The generated number will then correspond to a power divider in the set generated above, which is then used in the PDN instead of the ideal (target) power divider. This process is repeated until all 1023 power dividers in the PDN have been assigned the $S$-parameters corresponding to a non-ideal splitting ratio. Then, QZ field distribution is simulated 100 times.

Figs. $3 \mathrm{~b}$ and $3 \mathrm{e}$ show an example of the power and phase variations, respectively, in the QZ for one realization the power dividers selected using the described Monte Carlo method described above. From the abovementioned figures it can be seen that the symmetry observed on the plots corresponding to the ideal case is no longer present. Now, from the 100
QZ simulations we compute the maximum difference relative reference (MDRR) defined as

$$
\operatorname{MDRR}=\operatorname{MAX}_{x}-\operatorname{MIN}_{x}
$$

where $x$ can be either amplitude or phase. The MDRR is used to compare the performance of the QZ using ideal power splitters in [2] with the $100 \mathrm{MC}$ QZ simulations. Corresponding values for the MDRR of the amplitude and phase are reproduced in Table III. Here the figure-of-merit is recalculated for the ideal PWG of [2] for a QZ of $20 \times 20 \mathrm{~cm}^{2}$. In Figure 4 the cumulative distribution function (CDF) of the MDRR is shown for both the amplitude and the phase. Here, the MDRR is calculated over the entire QZ for each of the 100 QZ simulations. It can be seen from the figure that all 


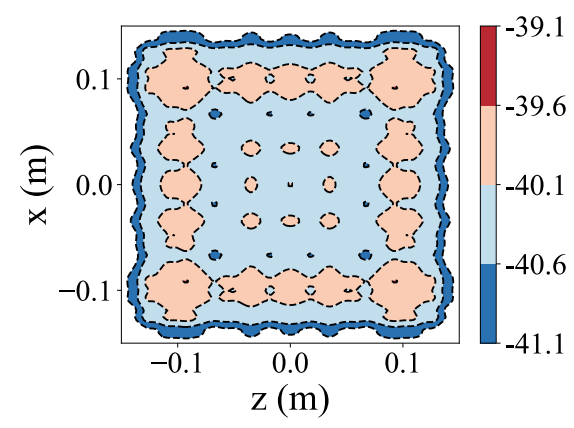

(a)

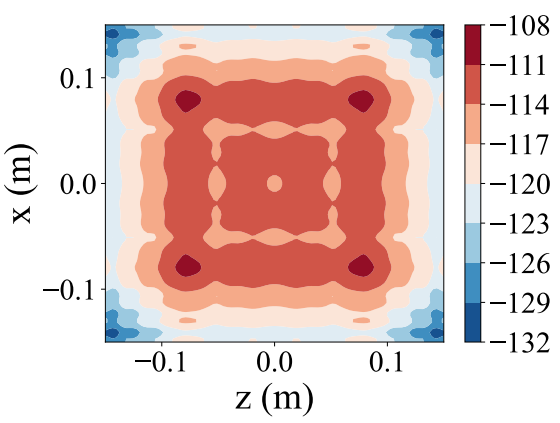

(d)

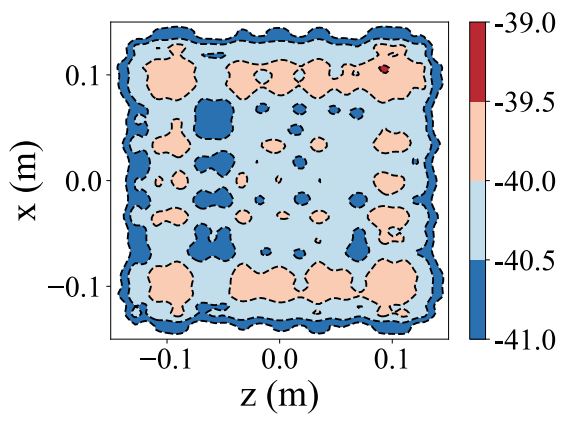

(b)

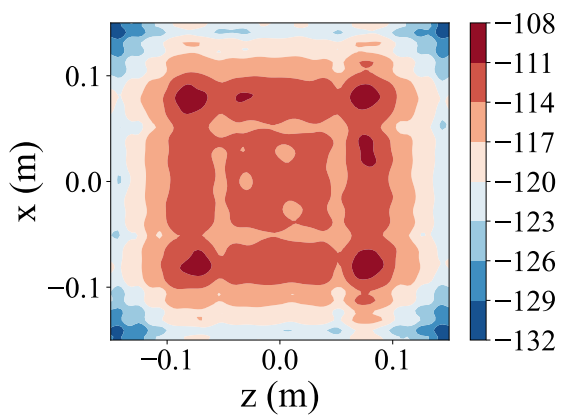

(e)

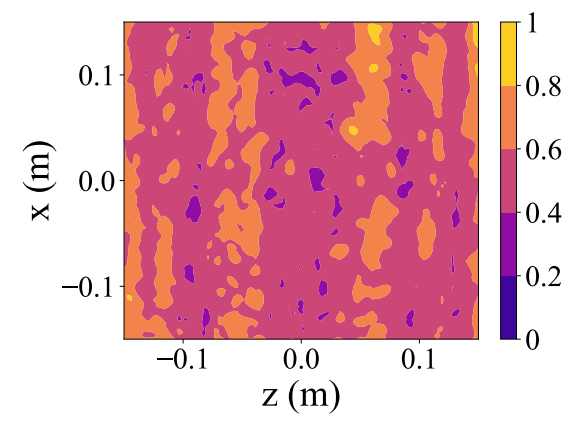

(c)

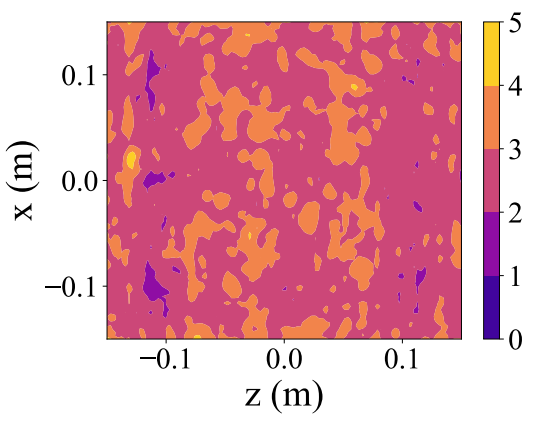

(f)

Fig. 3: Amplitude variation in the QZ at $28 \mathrm{GHz}$ obtained in (a) [2]. (b) shows an example of the QZ produced by power dividers designed in this paper and (c) shows the MDRR for each location in the QZ as a result of the Monte Carlo simulations. Corresponding results for the the phase variation in the QZ are shown in (d), (e) and (f), respectively.

TABLE III: QZ Performance

\begin{tabular}{c|c|c|c}
\hline \hline & Target & [2] & This work \\
\hline Diameter $\left(\mathrm{D}_{Q Z}\right)$ & $20 \mathrm{~cm}$ & $20 \mathrm{~cm}$ & $20 \mathrm{~cm}$ \\
\hline $\begin{array}{c}\text { Distance from PWG } \\
\text { to center of QZ }\end{array}$ & $45 \mathrm{~cm}$ & $45 \mathrm{~cm}$ & $45 \mathrm{~cm}$ \\
\hline Amplitude deviation & $\pm 1.0 \mathrm{~dB}$ & $\pm 0.53 \mathrm{~dB}$ & $\pm 0.91 \mathrm{~dB}$ \\
\hline Phase deviation & $\pm 5^{\circ}$ & $\pm 3.15^{\circ}$ & $\pm 4.79^{\circ}$ \\
\hline \hline
\end{tabular}

simulations, regarding both the phase and the amplitude, are below the target performance of Table III in the $20 \times 20 \mathrm{~cm}^{2}$ QZ.

Now, the MDRR is calculated for each location in the QZ, for both amplitude and phase. Thus, the MDRR shows the variation due to the MC simulations for each point in the QZ. Figs. 3c and 3f show the MDRR of each location in the QZ for the amplitude and phase respectively. From the abovementioned figures it can be seen that the MDRR of the amplitude is always below $1 \mathrm{~dB}$, while the MDRR of the phase is always below $5^{\circ}$.

\section{CONCLUSIONS}

The effect of manufacturing tolerances of waveguide power dividers on the quite zone of a plane wave generator has been examined. In order to simulate the variations, a normal distribution has been assumed for the design parameters of the power dividers. The non-ideal power dividers in the power distribution network introduce variations in the QZ, where the

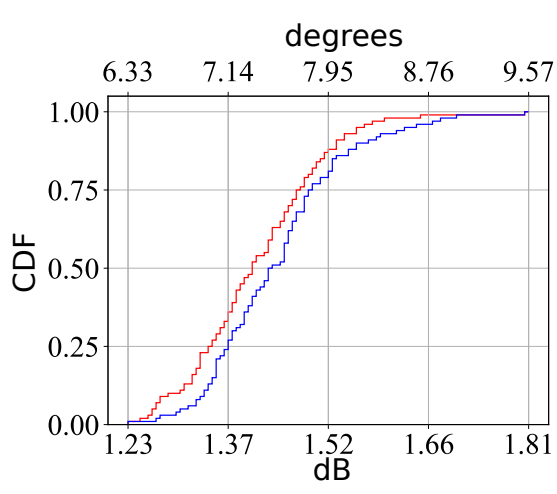

Fig. 4: The CDF of the MDRR for (blue) the amplitude and (red) the phase. Where the MDRR is calculated over the entire $20 \times 20 \mathrm{~cm}^{2}$ QZ for each of the 100 QZ simulations.

performance of the non-ideal PWG deviates from the ideal case. For the phase in the QZ, the variation is always within $\pm 4.8^{\circ}$ for each of the generated QZ. Similarly, the variation of the amplitude the variation is within $\pm 0.91 \mathrm{~dB}$. The variation is checked at each location in the QZ, which is found to be within $1 \mathrm{~dB}$ and $5^{\circ}$ for the amplitude and phase respectively. In future research the different power dividers have to be manufactured, measured and compared with the simulated values. The effect of the non-ideal power dividers at the edges of the frequency band of interest, 26.5- and $29.5 \mathrm{GHz}$, have 
to be evaluated as well.

\section{ACKNOWLEDGMENT}

The authors like to acknowledge Sara Catteau for the assistance with Python scripts and background material.

\section{REFERENCES}

[1] Radio Access Network; NR; User Equipment (UE) radio transmission and reception; Part2: Range 3 standalone, 3rd Generation Partnership Project (3GPP), Technical Specification (TS) 38.101-2, 06-2020, version 16.4 .0

[2] S. Catteau, M. Ivashina and R. Rehammar, "Design and Simulation of a $28 \mathrm{GHz}$ Plane Wave Generator for NR Measurements," 2020 14th European Conference on Antennas and Propagation (EuCAP), Copenhagen, Denmark, 2020, pp. 1-4, doi: 10.23919/EuCAP48036.2020.9135243.

[3] M. Poordaraee and A. A. Glazunov, "Plane Wave Synthesis with Irregular Chamber Planar Antenna Arrays for Compact OTA Measurements," 2019 13th European Conference on Antennas and Propagation (EuCAP), Krakow, Poland, 2019, pp. 1-5.

4] M. Poordaraee and A. A. Glazunov, "Chamber Array Antenna Layout for Compact OTA Measurements," 2020 14th European Conference on Antennas and Propagation (EuCAP), Copenhagen, Denmark, 2020, pp. 1-4, doi: 10.23919/EuCAP48036.2020.9135200.

[5] S. Catteau, "Design of a mm-Wave Plane Wave Generating Antenna Array for 5G Device Testing, Chalmers University of Technology, 2019.

[6] Yang, Songnan \& Fathy, Aly. (2009). Design Equations of Arbitrary Power Split Ratio Waveguide T-Junctions Using a Curve Fitting Approach. International Journal of RF and Microwave Computer-Aided Engineering. 19. 91 - 98. 10.1002/mmce. 20320.

division, Microwave Opt Tech Lett 14 (1997), 141-143

This paper's copyright is held by the author(s). It is published in these proceedings and included in any archive such as IEEE Xplore under the license granted by the "Agreement Granting EurAAP Rights Related to 